\title{
Genetically engineered nanocarriers for drug delivery
}

\author{
This article was published in the following Dove Press journal: \\ International Journal of Nanomedicine \\ 26 March 2014 \\ Number of times this article has been viewed
}

\author{
$\mathrm{Pu}$ Shi \\ Joshua A Gustafson \\ J Andrew MacKay \\ Department of Pharmacology and \\ Pharmaceutical Sciences, University \\ of Southern California, Los Angeles, \\ CA, USA
}

Correspondence: ] Andrew MacKay 1985 Zonal Avenue, PSC 306A, School of Pharmacy, University of Southern California, Los Angeles, CA 90089, USA Tel + I 323442 4I I8

Email jamackay@pharmacy.usc.edu

\begin{abstract}
Cytotoxicity, low water solubility, rapid clearance from circulation, and off-target side-effects are common drawbacks of conventional small-molecule drugs. To overcome these shortcomings, many multifunctional nanocarriers have been proposed to enhance drug delivery. In concept, multifunctional nanoparticles might carry multiple agents, control release rate, biodegrade, and utilize target-mediated drug delivery; however, the design of these particles presents many challenges at the stage of pharmaceutical development. An emerging solution to improve control over these particles is to turn to genetic engineering. Genetically engineered nanocarriers are precisely controlled in size and structure and can provide specific control over sites for chemical attachment of drugs. Genetically engineered drug carriers that assemble nanostructures including nanoparticles and nanofibers can be polymeric or non-polymeric. This review summarizes the recent development of applications in drug and gene delivery utilizing nanostructures of polymeric genetically engineered drug carriers such as elastin-like polypeptides, silk-like polypeptides, and silk-elastin-like protein polymers, and non-polymeric genetically engineered drug carriers such as vault proteins and viral proteins.
\end{abstract}

Keywords: polymeric drug carrier, non-polymeric drug carrier, gene delivery, GE drug carriers

\section{Introduction}

Drug-delivery systems are designed to lower toxicity and improve pharmacokinetic/ pharmacodynamic profiles of conventional drugs. ${ }^{1}$ Following intensive development by many groups, numerous drug carriers have been successfully developed. For the purposes of this review, we consider two general classes of drug carriers: chemically synthesized carriers, and genetically engineered carriers. ${ }^{2}$ Focusing on the latter, this manuscript compares these two different types of carriers in various aspects including features of the carriers, carrier synthesis, and cytotoxicity, for example. Significant progress has been made in the field of synthetic polymers to increase the efficiency of polymerization techniques and lower polydispersities. ${ }^{2,3}$ Despite this progress, genetic engineering provides unparalleled control over the component macromolecules used to build nanoparticulate carriers. ${ }^{4}$ This capability allows unique characteristics such as specific biodegradation profiles and fully customized polymer and nanocarrier architectures to be engineered and modified as needed for specific applications. Unlike chemically synthesized drug carriers, current research into genetically engineered carriers only scratches the surface of potential applications. During its emergence, it already produced multiple carriers that show unique potential for clinical application. Examples of such technologies include a highly potent yet side-effect-limiting doxorubicin formulation, ${ }^{5}$ a genetically engineered nanoparticle which effectively targets the coxsackievirus and 
adenovirus receptor, ${ }^{6}$ and naturally derived carrier proteins which entrap and allow delivery of hydrophobic drugs. ${ }^{7}$

Unlike chemically synthesized carriers, proteins offer unique opportunities to form nanostructures based on the well-established secondary, tertiary, and quaternary structures commonly found in natural proteins. Well studied secondary structures such as $\alpha$-helices and $\beta$-sheets can be used to bind together micro- or nanoparticle structures. ${ }^{8,9}$ In addition to unique structural opportunities, genetically engineered drug carriers have hierarchical structures, ${ }^{4,6}$ on which structurefunction studies might be accomplished by site-directed mutagenesis at the primary amino acid sequence. At the current time, genetically engineered drug carriers can be divided into two categories in the consideration of primary amino-acid sequences: 1) polymeric genetically engineered drug carriers, and 2) non-polymeric genetically engineered drug carriers. The relationship between vehicles for drug delivery, nanostructure formation, and protein polymers is visually conveyed in Figure 1, in which intersections 1, 2, and 3 represent polymeric drug carriers with nanostructures, non-polymeric carriers with nanostructures, and polymeric carriers without nanostructures, respectively.

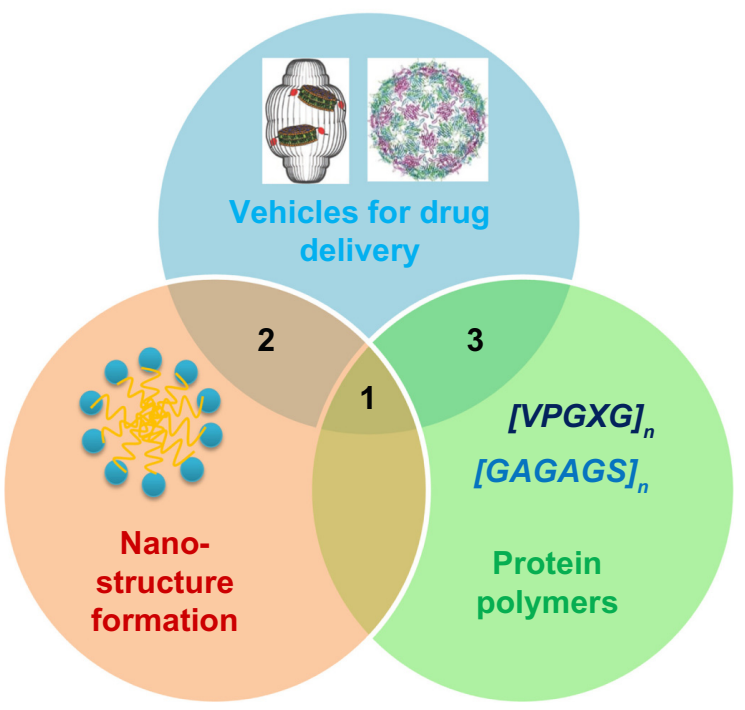

Figure I Design of genetically engineered drug carriers.

Notes: The field of biological nanomedicine (aka "BioNano") is emerging at the intersections between genetically engineered biomaterials, nano-assembly, and protein polymers. At intersection I, nanomedicines are being developed from protein polymers (eg, ELP, SLP, and SELP). At intersection 2, protein-based materials (eg, viral capsids and vault proteins) are being developed as platforms for assembly of nanostructures. At intersection 3, proteins that avoid structure formation (eg, intrinsically disordered proteins and XTEN fusion proteins) are being explored for their ability to alter biodistribution and efficacy.

Adapted with permission from Galaway FA, Stockley PG. MS2 viruslike particles: a robust, semisynthetic targeted drug delivery platform. Mol Pharm. 2013;10(I): 59-68. ${ }^{55}$ Copyright 2013 American Chemical Society, and Buehler DC, Toso DB, Kickhoefer VA, Zhou ZH, Rome LH. Vaults engineered for hydrophobic drug delivery. Small. 20II;7(I0):1432-1439. Copyright 20II WILEY-VCH Verlag GmbH \& Co. KGaA, Weinheim.

Abbreviations: ELP, elastin-like polypeptide; SLP, silk-like polypeptide; XTEN, extended recombinant polypeptide.
Examples of well-developed genetically engineered drug carriers include protein polymers composed from elastin-like polypeptides (ELPs), ${ }^{10}$ silk-like polypeptides (SLPs), ${ }^{11}$ extended recombinant polypeptide (XTEN) polymers, ${ }^{12}$ and silk-elastin-like polypeptides (SELPs).${ }^{13}$ Alternatively, nonpolymeric genetically engineered drug carriers with defined tertiary and quaternary structure have been developed from viral proteins ${ }^{14}$ and vault proteins. ${ }^{7}$ From the perspective of sequence-structure relations, genetically engineered drug carriers present varying macro-, micro-, or nanoscale properties, with differences in length and composition of amino-acid sequences. In this review, we primarily aim to provide a summary of polymeric and non-polymeric genetically engineered drug carriers, and focus on their drug-delivery applications using various nanostructures.

For the purposes of this review, a "polypeptide" is defined as a repetitive amino acid sequence built from a short motif. The term "protein polymer" is defined as an amino acid sequence that fulfills roles (eg, electrostatic or steric repulsion) filled by synthetic polymers. A protein polymer may (ELP) or may not (XTEN) be a polypeptide and may or may not produce secondary structure. In contrast, the term "protein" is defined as a non-repetitive amino acid sequence that generates tertiary and quaternary structures (vault and viral particles), which produce specific molecular functions.

\section{Genetically engineered polymeric drug carriers and their nanostructures}

Protein polymers consist of natural or unnatural repetitive amino-acid sequences and are generally biosynthesized in cells, either prokaryotes or eukaryotes. Because protein polymers can be engineered at the genetic level, their sequences can be precisely controlled. ${ }^{15-17}$ One significant advantage of protein polymers is that by changing several amino acids in the repetitive sequences, libraries of polymers with different charges, hydrophobicity, or secondary structures can be created to perform structure-function studies. ${ }^{15,16,18}$ Compared with conventional polymers, protein polymers may cause lower cytotoxicity, which may be due to the fact that they have biologically relevant mechanisms for proteolysis into relatively inert amino acids. ${ }^{16,19}$ Since 1986, when Ferrari et $\mathrm{al}^{20}$ reported the first protein polymer designed to be a potent drug carrier, a number of different protein polymers have been developed for use as drug carriers, such as ELPs, ${ }^{5,21-24}$ SLPs $^{25-27}$ and SELPs. ${ }^{28}$

\section{ELPs}

Elastin is a major extracellular matrix protein that provides resilience and elasticity in tissues and organs of many higher 
animals. ELPs are protein polymers which consist of repeats of amino acid sequence Val-Pro-Gly-Xaa-Gly ([VPGXG] $]_{\mathrm{n}}$ ), derived from a highly conserved repeat sequence in mammalian tropoelastin. ${ }^{10}$ In natural elastin, the guest residue $\mathrm{X}$ is frequently valine, alanine, or isoleucine. When the identity of $\mathrm{X}$ is changed in the context of ELPs, many interesting properties can be imparted and precisely tuned, for example, reversible phase-separations in aqueous solutions. ${ }^{2,15,29,30}$ One intriguing use of this guest residue-dependent modification of polymer properties has been the creation of ELP block copolymers, which have been constructed by genetically linking a hydrophobic block and a hydrophilic block together, for example, $[\text { VPGIG] }]_{\mathrm{n} 1}$-[VPGSG $]_{\mathrm{n} 2} \cdot{ }^{4}$ These block copolymers have been verified to form stable nanoparticle structures ranging from $50-90 \mathrm{~nm}$ in diameter, which have various functions in drug delivery, and the formation of which is dependent on the difference between the transition properties of the

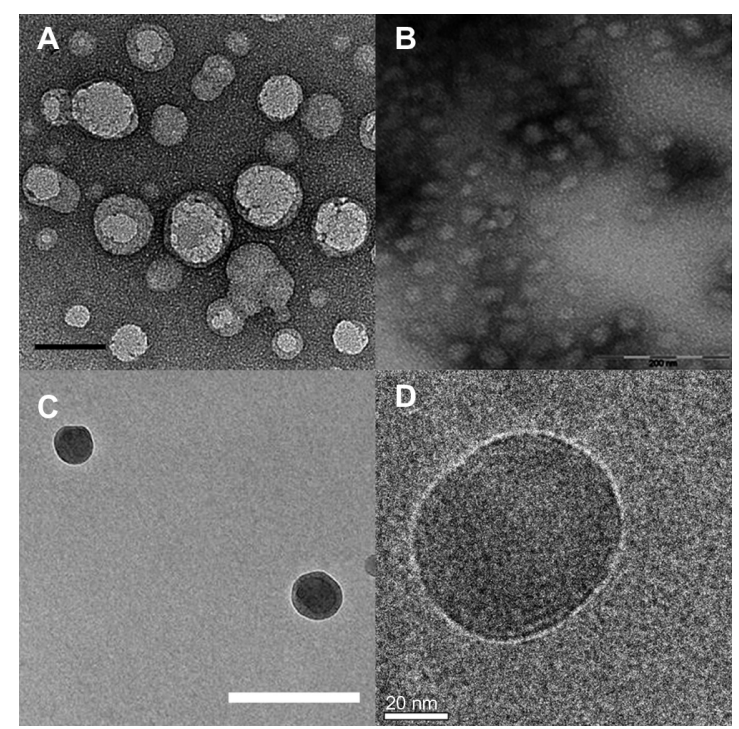

Figure 2 TEM of ELP nanoparticles. Diblock copolymers composed of ELPs with various guest residues assemble micelles.

Notes: (A) TEM image of A96196, which has a hydrophilic (Xaa = Ala, $n=96$, $\mathrm{N}$-terminus) and a hydrophobic ( $\mathrm{Xaa}=1 \mathrm{le}, \mathrm{n}=96, \mathrm{C}$-terminus) block. Scale bar $50 \mathrm{~nm}$. From Janib SM, Liu S, Park R, et al. Kinetic quantification of protein polymer nanoparticles using non-invasive imaging. Integr Biol (Camb). 2013;5(I):I83-194. ${ }^{23}$ Reproduced by permission of The Royal Society of Chemistry. (B) TEM image of ELP 196S96, which has a hydrophilic ( $\mathrm{Xaa}=\mathrm{Ser}, \mathrm{n}=96, \mathrm{C}$-terminus) and a hydrophobic (Xaa = lle, n=96, N-terminus) block. Scale bar 200 nm. From Janib SM, Pastuszka MF, Aluri S, et al. A quantitative recipe for engineering protein polymer nanoparticles. Polym Chem. 2014;5(5):1614-1625. ${ }^{4}$ Reproduced by permission of The Royal Society of Chemistry. (C) Cryo-TEM image of ELP E50160, which has a hydrophilic (Xaa = Val:Glu [4:I], n=50, N-terminus) and a hydrophobic (Xaa = lle, $n=60, C$-terminus) block. Scale bar $100 \mathrm{~nm}$. Reproduced with permission from García-Arévalo C, Bermejo-Martín JF, Rico L, et al. Immunomodulatory nanoparticles from elastin-like recombinamers: single-molecules for tuberculosis vaccine development. Mol Pharm. 2013;10(2):586-597.34 Copyright 2013 American Chemical Society. (D) CryoTEM image of ELP-96/90, which has a hydrophilic (Xaa = Val:Ala:Gly [1:8:7], $n=96$, $\mathrm{N}$-terminus) and a hydrophobic (Xaa $=\mathrm{Val}, \mathrm{n}=90, \mathrm{C}$-terminus) block. Scale bar $20 \mathrm{~nm}$. Reprinted with permission from Dreher MR, Simnick AJ, Fischer K, et al. Temperature triggered self-assembly of polypeptides into multivalent spherical micelles. J Am Chem Soc. 2008; I 30(2):687-694. ${ }^{33}$ Copyright 2008 American Chemical Society.

Abbreviations: ELP, elastin-like polypeptide; TEM, transmission electron microscopy; cryo-TEM, cryogenic transmission electron microscopy. hydrophilic and hydrophobic blocks. ${ }^{2,6,16,18,23,30-32}$ Figure 2 illustrates a series of ELP micelle nanoparticles formed by repetitive amino-acid sequences with different guest residues in hydrophobic and hydrophilic blocks. ${ }^{4,23,33,34}$

\section{SLPs}

Silk proteins are natural polymers produced by either silkworms or spiders. Silkworm silk fibroin from Bombyx mori and spider silk fibroin from Nephila clavipes are two of the most well studied silk proteins at present. ${ }^{35,36}$ They generally are considered to be block copolymers with highly conserved repeats of short side-chain amino acids as hydrophobic blocks and short sequences of larger side-chain or charged amino acids as hydrophilic blocks., ${ }^{2,37}$ The most common amino-acid sequence of SLPs derived from Bombyx mori silkworms is the $[\mathrm{GAGAGS}]_{\mathrm{n}}$ repeat, while the most common spider silk SLP is [GRGGLGGQGAGAAAAAGGAGQGGYGGLGS QG] $]_{n}$, derived from Nephila clavipes. With the incorporation of cationic polylysine and/or polyarginine deoxyribonucleic acid (DNA)-binding domains, nanofibers, and nanoparticles formed by SLPs have been successfully applied to the field of gene delivery. Spider silk-based nanoparticles containing tumor-homing peptides such as F3 (KDEPQRRSARLSAKPA PPKPEPKPKKAPAKK), Lyp1 (CGNKRTRGC), and CGKRK and poly(L-lysine) domains have been demonstrated to deliver target-specific plasmid DNA (pDNA) to the tumor cells (MDAMB-435 and MDA-MB-231) with low cytotoxicity and high efficiency. Therefore, these nanoparticles may have potential to be utilized as DNA carriers in cancer gene therapy. ${ }^{26,37}$

\section{SELPS}

SELPs have both motifs from the silkworm silk sequence [GAGAGS $]_{\mathrm{n}}$ and mammalian tropoelastin sequence [VPGVG] $n$. Because the silk blocks of SELPs tend to form $\beta$-sheet structures with intensive inter- and intramolecular hydrogen-bond interactions, SELPs with high silk content precipitate out of aqueous solution at relatively low concentration. ${ }^{17}$ However, with the increasing repeat number of elastin blocks that disorder the formation of crystalline silk structures, the entire SELP block becomes water soluble at low temperature. This property is critical for protein polymer purification and drugdelivery formulation. ${ }^{38}$ SELP hydrogels are formed after an irreversible phase transition, which makes them amenable to the development of solvent-free injectable depots. As such, SELPS are one of the most exciting emerging carriers for drugs and gene therapy. ${ }^{39}$ It has been speculated that the formation of a micelle core by hydrophobic interactions of the silk blocks is the driving force to assemble SELP nanoparticle structures that have potential drug-delivery applications. ${ }^{28}$ 


\section{Other polymeric genetically engineered drug carriers}

Besides ELPs, SLPs, and SELPs, there are many other types of polymeric genetically engineered drug carriers. For example, Farmer and Kiick ${ }^{40}$ have created alanine-rich helical proteins. This repeating helical protein, which contains glutamine and glutamic acid, can form nanofibril structures and is being developed as a multivalent drug nanocarrier. ${ }^{40}$ Amunix Inc., a biotechnology company focused on protein polymers, has genetically engineered a long protein polymer termed XTEN, which is composed of hydrophilic and negatively charged residues. It has been demonstrated that the half-life of many protein therapeutics can be drastically increased by attaching XTEN, and the half-life can be tuned by varying the length of the XTEN polymer. ${ }^{12}$ The exact mechanism of how XTEN increases protein half-life has not been fully delineated; however, it is plausible that slow proteolytic biodegradation, high molecular weight, and anionic electrostatics repel the extracellular matrix in the glomerulus. By reducing the rate of renal clearance, XTEN polymers may prolong protein half-life similarly to a synthetic polymer (eg, polyethylene glycol, HPMA [N-(2-hydroxypropyl) methacrylamide], and dextran).

\section{Polymeric nanocarriers that mediate drug delivery}

Protein polymers such as ELPs, SLPs, and SELPs can form nanoparticle structures under certain conditions. In the last 2-3 years, multiple articles have been published focusing on these nanocarriers in the delivery of genes and drugs. ${ }^{6,26,27,29,31,41-43}$

\section{ELP-mediated drug delivery}

In the ELP field, Sun et $\mathrm{al}^{6}$ utilized fusion protein technology to decorate the corona of an ELP block copolymer G[VPGSG] ${ }_{48}{ }^{-}$ $\left.{ }_{\mathrm{VPGIG}}\right]_{48}$ with several different useful proteins. The attachment of the knob domain of adenovirus serotype 5 fiber protein to the serine block of this polymer was one example of this technology. Using dynamic light scattering, the genetically modified knob-ELP fusion protein was measured to form nanoparticles with a $\sim 40 \mathrm{~nm}$ diameter with the knob domain on the surface. Cellular uptake studies using a coxsackievirus and adenovirus receptor-expressing hepatocyte cell line revealed that the knob-ELP fusion protein presented significantly stronger colocalization to lysosomes inside hepatocytes than plain ELPs, which indicated that the knob domain of adenovirus serotype 5 fiber protein was the critical factor to facilitate targeted cellular internalization of the fusion protein nanoparticles. ${ }^{6}$ Moreover, FKBP (FK506-binding protein), the cognate receptor of an antiproliferative drug rapamycin (Rapa) has also been genetically fused onto the corona of micelles assembled from the ELP block copolymer (Figure 3A). ${ }^{41,42}$ Because of high-avidity binding of the drug to the receptor, the new fusion protein (FKBP-ELP [FSI]) slowed the terminal half-life of drug release to 57.8 hours (Figure 3B). ${ }^{42}$ The in vivo antitumor and immunosuppressant applications of the new Rapa formulation (FSI-Rapa) were respectively examined in a MDA-MB-468 breast cancer xenograft nude mouse model

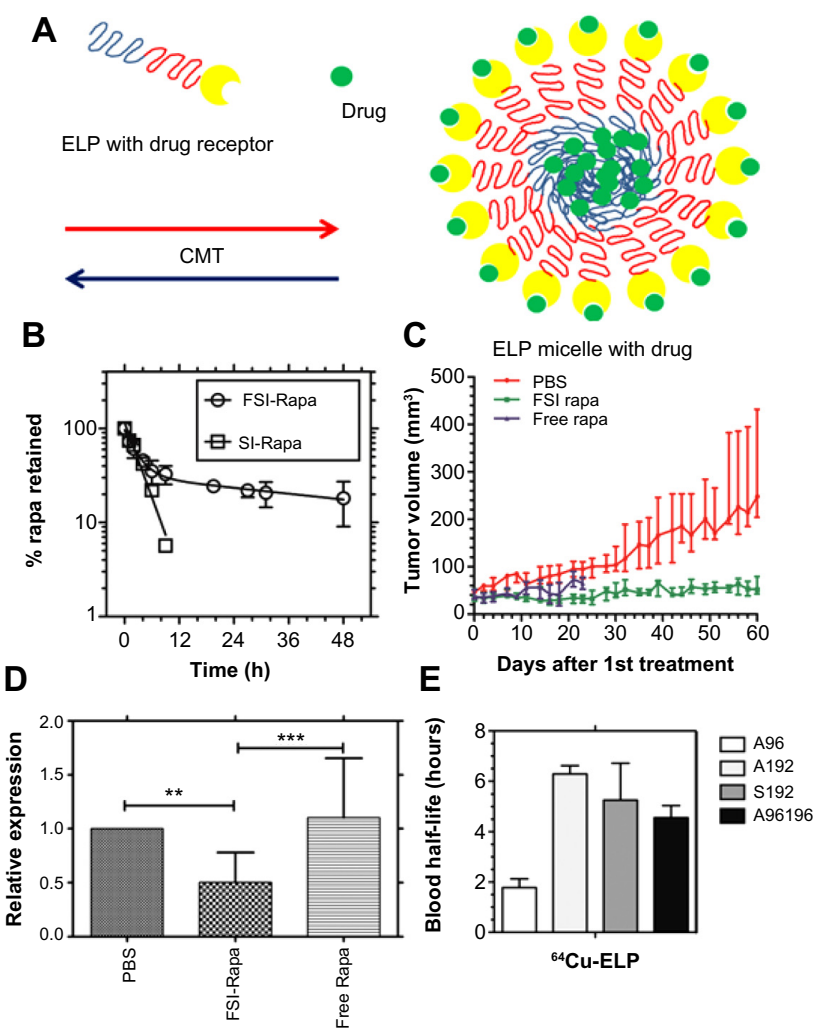

Figure 3 Rapa encapsulated by FKBP-decorated nanoparticles has both anticancer and immunosuppressive efficacy.

Notes: (A) ELP nanoparticles fused genetically to the cognate receptor of RapamycinFKBP can specifically carry the drug with high avidity. (B) FSI significantly prolongs drug release compared with plain ELP (SI). (C) FSI-Rapa has lower cytotoxicity and greater antitumor efficacy than free Rapa in an MDA-MB-468 breast tumor xenograft mouse model. Compared with free Rapa group, which showed severe cytotoxicity (15\% bodyweight loss by day 23), no obvious systemic cytotoxicity was observed in the FSI-Rapa group. (A), (B), (C) Reproduced from J Control Release, I7I(3), Shi P, Aluri S, Lin YA, et al. Elastin-based protein polymer nanoparticles carrying drug at both corona and core suppress tumor growth in vivo, 330-338, ${ }^{42}$ Copyright 2013, with permission from Elsevier. (D) FSI-Rapa suppresses transcription and expression of the protease cathepsin-S (CATS), a biomarker of lacrimal gland autoimmune dacryoadenitis, better than free Rapa in a mouse model of Sjögren's syndrome. Reproduced from J Control Release, I7I(3), Shah M, Edman MC, Janga SR, et al, A rapamycin-binding protein polymer nanoparticle shows potent therapeutic activity in suppressing autoimmune dacryoadenitis in a mouse model of Sjogren's syndrome, 269-279,43 Copyright 2013 with permission from Elsevier. (E) The blood half-lives of four ELPs estimated by pharmacokinetic modeling in mice based on micro-PET imaging. ***indicates a $P$-value of $<0.001$; **indicates a $P$-value of $<0.01$ by one-way ANOVA with Tukey's multiple comparison test. From Janib SM, Liu S, Park R, et al. Kinetic quantification of protein polymer nanoparticles using non-invasive imaging. Integr Biol (Camb). 2013;5(I):183-194. ${ }^{23}$ Reproduced by permission of The Royal Society of Chemistry.

Abbreviations: CMT, critical micelle temperature; ELP, elastin-like polypeptide; FKBP, FK506-binding protein; FSI, FKBP-ELP; PBS, phosphate buffered saline; PET, positron emission tomography; Rapa, rapamycin; SI, ELP S48I48. 
and Sjögren's syndrome non-obese diabetic mouse model. It was discovered that FSI-Rapa showed not only significantly less cytotoxicity but greater efficacy in tumor regression and autoimmune response suppression than the free drug, respectively, in the two models (Figure $3 \mathrm{C}$ and D). ${ }^{42,43}$ In addition, the blood half-lives of ELPs in mice were estimated by a multi-compartmental pharmacokinetic model using the data from noninvasive micro-PET (positron emission tomography) imaging. Depending on molecular weight and assembled structure, the blood half-lives of ELPs vary from 2 to 6 hours in vivo (Figure 3E) ${ }^{23}$ Because the half-life of Rapa release is much longer than the blood half-lives of ELPs, it has been speculated that the drug will remain associated with the carrier blood circulation with minimal detachment, which may reduce systemic side effects.

The Simnick et $\mathrm{al}^{31}$ study employed the same fusion protein technology to genetically revise the corona of ELP amphiphilic block copolymers with the NGR (Asn-Gly-Arg) tripeptide ligand targeting the CD13 receptor. NGR-decorated ELP amphiphilic block copolymers were designed to competently target CD13 receptors expressed highly in tumor vasculature and perivascular cells. The results showed NGR-decorated ELP amphiphilic block copolymers formed particles 25-30 nm in radius above the critical micelle temperature, and NGR micelles achieved greater vascular retention and extravascular accumulation in tumor tissue compared with normal tissue in an intravital laser scanning confocal fluorescence microscopy study. ${ }^{31}$ These successful studies obviously reveal the advantages of using protein polymer nanocarriers such as ELPs precise molecular modification at the genetic level. It remains challenging to chemically attach a complex protein such as a drug receptor to synthetic carriers; however, using protein fusion technology, the modification on genetically engineered drug carriers can be completed seamlessly.

Another example of ELP-mediated drug delivery using nanoconstructs is elastin-mimetic amphiphilic diblock copolymer. These materials are based on the sequence $[(\mathrm{VPGVG})(\mathrm{VPGEG})(\mathrm{VPGVG})(\mathrm{VPGEG})$ $(\mathrm{VPGVG})]_{10-}\left(\left[\mathrm{Glu}_{2}\right]_{10}\right)$ as the hydrophilic block and $\left.\left[(\text { IPGVG })_{2} \text { VPGYG(IPGVG) }\right]_{2}\right]_{15-}\left([\mathrm{Tyr}]_{15}\right)$ as the hydrophobic block. This block copolymer has been confirmed to form stable micelles and efficiently solubilize and encapsulate dipyridamole, a model drug with anti-inflammatory activity. In vitro and in vivo drug release experiments have verified that the retention time of dipyridamole inside the micelle core reduces with the decrease of the length of the hydrophobic block repeats. It also has been discovered that dipyridamole encapsulation effectively suppresses in vivo recruitment of neutrophils in the presence of an inflammatory stimulus. ${ }^{29}$

\section{SLP-mediated drug delivery}

There are a number of SLP-based materials being developed as nanocarriers for gene delivery. Numata, MieszawskaCzajkowska et $\mathrm{al}^{26}$ and Numata, Reagan et $\mathrm{al}^{27}$ have employed natural silk sequence to fabricate genetically engineered silklike recombinant protein including poly(L-lysine) domains and tumor-homing peptides (THPs). pDNA can interact with poly(L-lysine) domains of the silk-like recombinant protein and together form globular pDNA-silk nanocomplexes with diameters from 100 to $250 \mathrm{~nm}$. So far, four different types of THPs (F3, Lyp1, monomeric CGKRK, and dimeric CGKRK) have been genetically engineered to two different types of silk-like proteins. MDA-MB-435 melanoma cells and highly metastatic human breast tumor MDA-MB-231 cells were used to test the binding of THP-targeted pDNA-silk nanocomplexes to specific tumorigenic cells. In vitro and in vivo transfection experiments have demonstrated that all types of pDNA-silk nanocomplexes with different THPs (F3, Lyp1, monomeric CGKRK, and dimeric CGKRK) have specifically targeted tumorigenic cells, while no targeting was found when MCF10A non-tumorigenic mammary breast epithelial cells were used. Field emission scanning electron microscopy was applied to investigate the mechanism of the specificity of pDNA-silk nanocomplexes to tumorigenic cells. pDNA-silk nanocomplex with F3 THP was observed to be absorbed through the surface of MDA-MB-231 cells but not through MCF10A cells, which indicated that the targeting specificity might be caused by THPtumor cell surface receptor-mediated absorption. ${ }^{26,27}$

\section{SELP-mediated drug delivery}

SELPs have previously been used to form hydrogels that are used for localized gene delivery. ${ }^{44}$ So far, no study has been published reporting direct SELP nanoparticle-based drug delivery. However, efforts have been made to synthesize many promising SELP nanostructures that are potentially excellent carriers for different drugs. Recently, Xia et $\mathrm{al}^{28}$ reported different structures such as nanoparticles, hydrogels, and nanofibers could be reversibly or irreversibly assembled by precisely tuning the ratio of silk to elastin of SELPs. As illustrated in Figure 4A, three different SELPs (SE8Y, S2E8Y, and S4E8Y) were biosynthesized with various silk to elastin ratios, and their morphological changes upon heating to $60^{\circ} \mathrm{C}$ were studied by atomic force microscopy (Figure 4B). At $60^{\circ} \mathrm{C}$, both SE8Y and S2E8Y assembled spherical nanoparticles with a hydrodynamic radius of $241 \pm 13$ and $212 \pm 16 \mathrm{~nm}$, respectively; however, the silk blocks of S4E8Y underwent crosslinking to form gel states instead of obvious nanoparticles. Distinct structures were observed in the aqueous solutions of the three SELPs in the cooling-down process (Figure 4C). At 


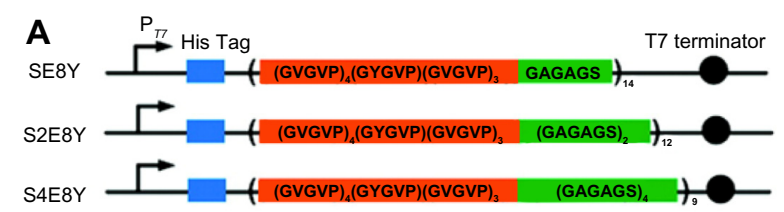

B
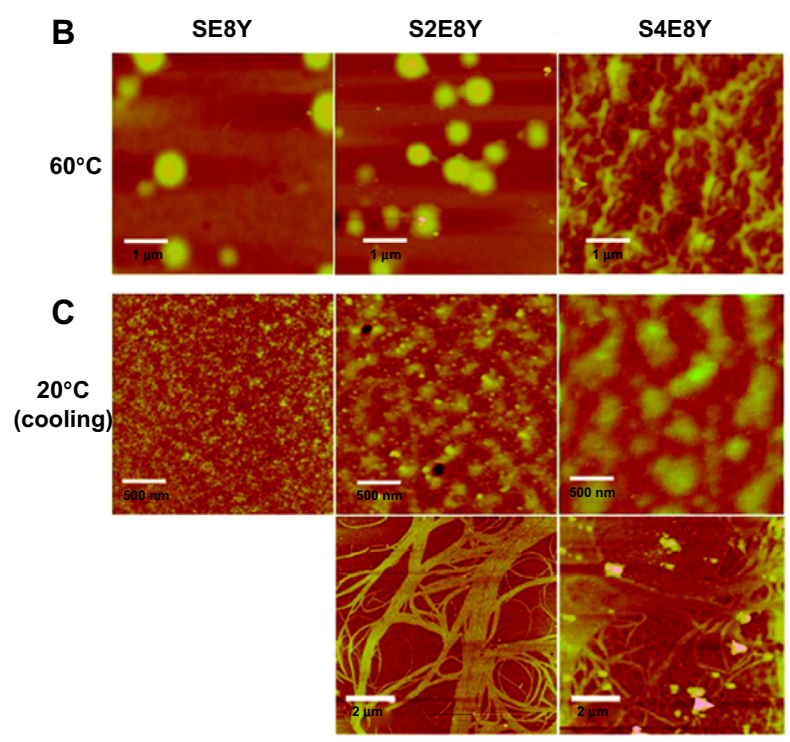

Figure 4 Silk-elastin-like protein polymers with different ratios of silk to elastin self-assemble into various nanostructures.

Notes: (A) SELP constructs SE8Y, S2E8Y, and S4E8Y, which contain varying ratios of the silk-to-elastin blocks in each monomer repeat. (B and $\mathbf{C}$ ) Atomic force microscopy images present the nanostructures self-assembled from SE8Y, S2E8Y, and S4E8Y at $60^{\circ} \mathrm{C}(\mathrm{B})$ and $20^{\circ} \mathrm{C}(\mathrm{C})$.

Adapted with permission from Xia XX, Xu Q, Hu X, Qin G, Kaplan DL. Tunable self-assembly of genetically engineered silk - elastin-like protein polymers. Biomacromolecules. 20 II;I2(II):3844-3850. ${ }^{28}$ Copyright 201 I American Chemical Society.

Abbreviation: SELP, silk-elastin-like polypeptide.

$20^{\circ} \mathrm{C}$, small micelle-like nanoparticles were observed in SE8Y solution, worm-like nanostructures composed of small spherical particles were assembled by S2E8Y, and large, polydisperse aggregates appeared in S4E8Y solution. Moreover, aligned nanofibers by the crosslinking of the silk blocks were also observed for S2E8Y and S4E8Y. The study demonstrated the formation of various nanostructures self-assembled by SELPs which may be potentially used as nanocarriers for controlled drug delivery. ${ }^{28}$ Another advantage of genetically engineered carriers over chemically synthetic carriers is that structureactivity studies can be easily and accurately performed with modifications in primary amino-acid sequences. By changing DNA sequences, various SELPs can be biosynthesized with different elastin to silk ratios. ${ }^{28}$

\section{Non-polymeric drug carriers and their structures}

Non-polymeric proteinaceous drug carriers are characterized by their lack of repetitive amino-acid sequences, and thus rely on the formation of self-assembled quaternary structures to act as drug carriers. To date, there are many non-polymeric genetically engineered drug carriers that have been developed and applied extensively in gene and drug delivery. ${ }^{7,14,45-49}$ In this review of nanometer-scale drug carriers, we highlight two main categories of non-polymeric genetically engineered drug carriers that form useful nanostructures: vault proteins and viral proteins.

\section{Vault protein}

About 25 years ago, vault protein was discovered as the most bulky ribonucleoprotein complex, with a size of 13 MDa. ${ }^{50}$ Vault protein itself forms $71 \mathrm{~nm} \times 42 \mathrm{~nm} \times 42 \mathrm{~nm}$ nanoparticles and is abundant and conserved in most eukaryotes. ${ }^{50,51}$ Previous studies have revealed the broad cellular functions of vault protein, including nuclear-cytoplasmic transport, mRNA (messenger ribonucleic acid) localization, drug resistance, cell signaling, nuclear pore assembly, and innate immunity. ${ }^{52,53}$ The large ribonucleoprotein complex consists of three different types of proteins: major vault protein (MVP), vault poly(adenosine diphosphate-ribose) polymerase, and telomerase associated protein 1. Because MVP comprises $75 \%$ of the native vault protein mass and is sufficient to form vault nanoparticles on its own, in most studies only MVP was expressed. ${ }^{7}$ Vault nanoparticles are regarded to be promising drug carriers because they 1) have a spacious internal volume $\left(5 \times 10^{4} \mathrm{~nm}^{3}\right)$, which is adequate for the encapsulation of bioactive molecules; 2) consist of naturally occurring amino-acid sequences which have no known cytotoxicity and immunogenicity and are easy to modify at the genetic level; and 3) form a "dynamic" nanostructure which can dissociate into halves in a low $\mathrm{pH}$ environment, improving their utility for drug release. . $^{7,45,46,54}$

\section{Viral proteins}

Viral nanoparticle assemblies are another prevalent strategy in the field of non-polymeric nanocarriers for drug delivery. Naturally, viruses can infect plants and animals effectively and transfer their genetic materials (DNA, RNA, or proteins) to the host cells. ${ }^{47}$ Virus-like particles (VLPs) take advantage of this highly evolved and efficient transfer strategy to deliver their cargos by mimicking the natural process of viruses. VLPs have their own advantages: 1) milligram quantities of VLPs can be produced quickly and efficiently, which allows easy scale-up; 2) VLPs tend to be very robust because of their protein capsids and are stable in a range of solvents; and 3) VLPs possess great cell membrane penetration ability because of the viral features. Bioconjugations 
and encapsulations have been performed on VLPs to achieve decent gene and drug delivery in many studies. . $^{14,47-49}$

\section{Drug delivery using non-polymeric protein nanoparticles}

Non-polymeric genetically engineered drug carriers such as vault protein and viral proteins have nanometer-range structures. They have their own advantages serving as drug nanocarriers. According to recent publications, many modifications over their primary sequences of these nanocarriers have been accomplished to improve their capability to specifically target disease cells or to efficiently encapsulate bioactive molecules. ${ }^{7,45-48,55,56}$

\section{Vault protein mediated drug delivery}

Kar et $\mathrm{al}{ }^{45}$ have been exploring vault protein-mediated drug delivery using several different strategies. Recently, they have successfully loaded CCL21, a lymphoid chemokine into vault nanostructures. CCL21 can naturally bind to CCR7, a cellular chemokine receptor. Therefore, CCL21 can attract cells that highly express CCR7 such as dendritic cells, naïve and memory T-cells, and natural killer and natural killer T-cells to effectively kill cancer cells. ${ }^{45}$ CCL21 has been efficiently encapsulated into vault nanoparticles by genetically fusing onto a vault-targeting domain named INT (for vault INTeraction). It was discovered that the administration of CCL21INT-Vault complex into lung cancer mice enhanced the recruitment of leukocytic infiltrates, inhibited tumor growth, and reduced immune-suppressive cell frequencies. ${ }^{45}$

Another example of vault protein technology was the use of nanodisk (ND) nanoparticles to construct ND-INT complexes (NDI) to facilitate the encapsulation of therapeutics into vault nanoparticles (Figure 5A), ${ }^{7,45,46}$ NDs were $\sim 10-20 \mathrm{~nm}$ lipid nanoparticles consisting of small discoidal lipid bilayer fragments derived from apolipoprotein-AI. With the genetic fusion of INT to ND, NDI acquired the ability to entrap a wider range of therapeutics into vault particles than standard INT. ${ }^{7}$ A gene transcription regulator, all-trans retinoic acid (ATRA), which bound to the retinoid acid receptor and retinoid $\mathrm{X}$ receptor and altered functional genes on proliferation, differentiation, and apoptosis was tested and demonstrated to be encapsulated into NDI-Vault nanocomplex (Figure 5B). ATRA bioactivity in NDI-Vault nanocomplexes was also confirmed by MTT (3-(4,5-dimethylthiazol-2-yl)-2,5-diphenyltetrazolium bromide) assay in a malignant hepatoma cell line (HepG2) showing that NDI-Vault complexed with ATRA decreased $42 \%$ of HepG2 cell viability, while free ATRA induced only $18 \%$ cell death (Figure $5 \mathrm{C}$ ). ${ }^{7}$ The results demonstrate the

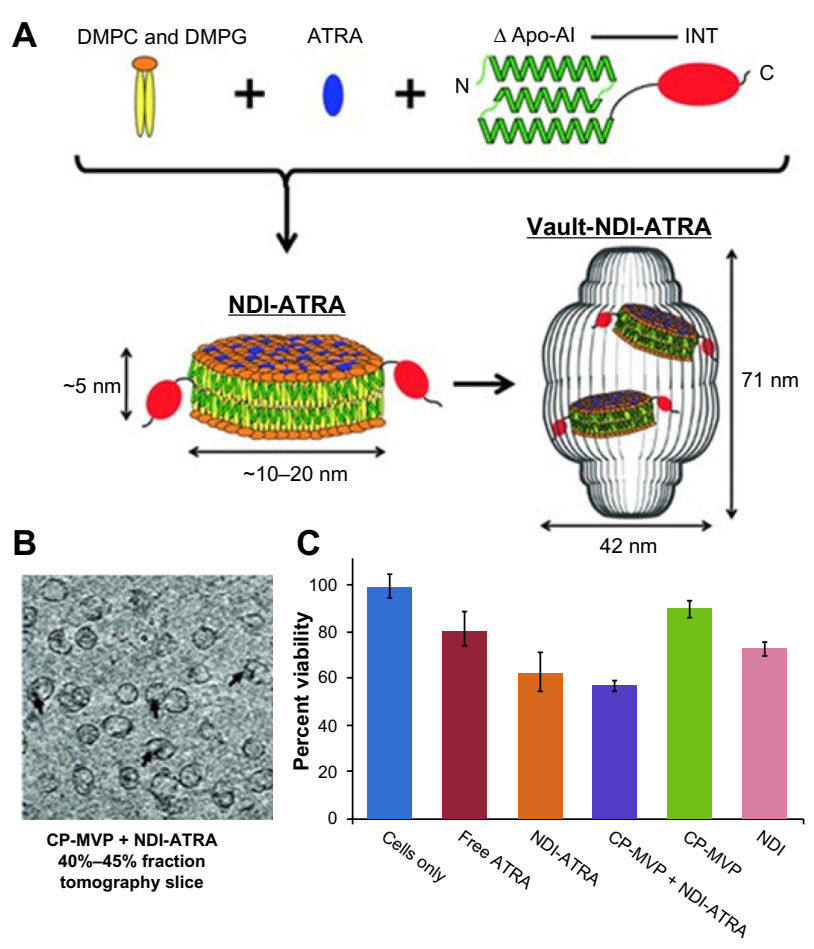

Figure $\mathbf{5}$ Vault protein engineered for hydrophobic drug delivery.

Notes: (A) Schematic diagram representing NDI-ATRA formation and encapsulation by the vault nanoparticle. Components are not drawn to scale. (B) CP-MVP + NDIATRA electron microscopic tomography slice showing NDI-ATRA vault packaging. (C) HepG2 cell viability assay. NDI-ATRA and CP-MVP + NDI-ATRA both display increased toxicity than free ATRA over the course of $I 20$ hours.

Adapted with permission from Buehler DC, Toso DB, Kickhoefer VA, Zhou ZH, RomeLH.Vaults engineered forhydrophobic drug delivery. Small. 20 I I;7(I0): 1432-I439.7 Copyright 201 I WILEY-VCH Verlag GmbH \& Co. KGaA, Weinheim.

Abbreviations: $\triangle \mathrm{Apo}-\mathrm{Al}$, a truncated form of apolipoprotein-Al (Apo-Al, amino acids 44-200); ATRA, all-trans retinoic acid; CP-MVP, the purified vaults; DMPC, dimyristoylphosphatidylcholine; DMPG, dimyristoylphosphatidylglycerol; INT, vaulttargeting domain; NDI, nanodisk-INT complex.

highly insoluble hydrophobic drug ATRA can be efficiently packaged into NDI-Vault complex and remain biologically active. Compared with the free drug that is cleared off rapidly, ATRA is slowly released from the dynamic vault structure and achieves greater drug efficacy. ${ }^{7}$

\section{Viral protein-medicated drug delivery}

Yildiz et $\mathrm{al}^{47}$ employed viral nanoparticles from a plant virus named cowpea mosaic virus (CPMV) to accomplish efficient intracellular delivery. In their study, the surface of CPMV nanoparticles was modified with polyarginine (R5) cell-penetrating peptides using bioconjugation techniques with the help of a hydrazone linker. Cell uptake efficiency was examined using both CPMV-R5 VLPs and plain CPMV particles. The result demonstrated that CPMV-R5 could be more efficiently taken up by a human cervical cancer cell line (HeLa) than plain CPMV particles. The result also indicated that higher R5 peptide density on the surface of CPMV-R5 particles determined greater efficiency of HeLa 
cell uptake. The surface cell-penetrating peptide modification also altered intracellular trafficking of CPMV nanoparticles. It was observed that plain CPMV particles were mainly trapped in endolysosomes after cell uptake, while 30\%-50\% of CPMV-R5 nanoparticles escaped from the endosome and trafficked to other cellular compartments. ${ }^{47,48}$ This finding provided CPMV-R5 nanoparticles with a promising future to encapsulate bioactive molecules and deliver them to different compartments within the cytoplasm.

There are two recent studies focusing on small interfering RNA (siRNA) delivery using engineered viral nanoparticles. ${ }^{55,56}$ Galaway and Stockley ${ }^{55}$ assembled a new VLP with the RNA bacteriophage MS2 coat protein and an RNA conjugate of an siRNA and a capsid assembly signal. The nanoparticle efficiently entered HeLa cells via receptor-mediated endocytosis and had significant siRNA effects at nanomolar concentrations. ${ }^{55}$ The study conducted by Choi et $\mathrm{al}^{56}$ utilized a capsid shell, integrin-targeting peptide, and p19 RNA-binding protein to assemble a nanocarrier for siRNA delivery. The capsid nanocarriers had affinity both for siRNA on the interior and cellular integrin on the exterior. It was discovered that RGD (Arg-GlyAsp) peptides on the surface enabled the capsid nanoparticles to target cancer cells that had high $\alpha_{\mathrm{v}} \beta_{3}$ integrin expression and deliver siRNA to the cytosol of the targeted cells. ${ }^{56}$ In both studies, engineered VLPs protected siRNA from the external nucleases and facilitated the endocytosis of the entire nanocarriers with the payload of siRNA. Besides the precise and seamless modification at the genetic level, genetically engineered carriers have another advantage over chemically synthetic carriers, which is the capability to produce large quantities of identical carriers by a one-step biosynthesis. Carriers genetically engineered from both vault protein and viral proteins can be scaled up from the milligram to gram scale relatively quickly, which is not always easy to accomplish using multistep formulations of nanoparticles prepared from synthetic materials.

\section{Discussion}

As an emerging class of efficient drug carriers, genetically engineered nanocarriers have been successfully evaluated in the delivery of a large number of drugs. Potential biocompatibility and controlled immunogenicity make them attractive technologies compared with chemically synthesized carriers; however, significant work remains to be done in this area ${ }^{57}$ Urry et $\mathrm{al}^{58}$ tested a $\gamma$-irradiated ELP monoblock (VPGVG) $_{\sim 120}$ and discovered no acute systemic toxicity in mice (intraperitoneal and intravenous), no systemic antigenicity in guinea pigs (intravenous). Moreover, it was found that subcutaneously injected ELPs could not generate antibodies unless complete Freund's adjuvant was added. ${ }^{57,59}$ Similarly to ELPs, Cappello et al ${ }^{38}$ also found low immunogenicity of a SELP with the sequence of $\left[(\text { GVGVP })_{8}(\text { GAGAGS })_{2}\right]_{18}$ when evaluated in rabbits. The SELP polymer was injected at time zero $(10 \mathrm{mg}), 6$ weeks $(0.5 \mathrm{mg})$, and 8 weeks $(0.5 \mathrm{mg})$ without adjuvant, and then sera samples were tested by enzyme-linked immunosorbent assay after 9 weeks. No reactivity (titer $<2$ ) of the samples was discovered for binding to pure (VPGVG) sequence. On the contrary, sera samples collected from SELP with complete Freund's adjuvant group showed a serum titer of 480-fold. ${ }^{57,59}$ As these materials move towards translational studies, more tests are needed to demonstrate the safety of these genetically engineered nanocarriers in vivo. Thus far, published data reveal that no antibody response has been stimulated with the administration of these protein polymers alone, suggesting that genetically engineered nanocarriers may be promising platforms for the development of new drug-delivery systems. ${ }^{57}$

Recombinant protein fusion technology is one of the major advantages in genetically engineered nanocarrier delivery and has been widely used in many applications. ${ }^{31,42,43}$ Compared with chemically synthesized carriers that require complicated and low-efficiency chemical conjugation reactions with many byproducts, genetically engineered nanocarriers utilize fast and efficient molecular cloning technique to link the drug or other functional domains onto the carriers at the DNA level. When biosynthesized, the resulting fusion products have nearly perfect homogeneity and monodispersity in large ( $\mathrm{mg}$ to g) quantities. ${ }^{6,42}$ Drugs with few modification sites, low stability in organic solvents, and/or poor chemical conjugation efficiency are amenable to delivery by genetically engineered nanoparticles. Furthermore, Shi et $\mathrm{al}^{42}$ reported a small screening assay for suitable drug molecules for ELP micelle encapsulation and found that drug molecules with high hydrophobicity (log $P$-values) and/or large numbers of hydrogen-bond donors and acceptors had higher ELP encapsulation efficiency than others. Therefore, drugs that are currently difficult to formulate using more conventional delivery vehicles might be good candidates for delivery by genetically engineered nanoparticles.

\section{Conclusion}

Recently, major innovations in the field of drug delivery have resulted from advancements in the use of genetic engineering to biosynthesize genetically engineered biological nanocarriers, which were the focus of this review. The advantages of genetically engineered carriers over chemically 
synthesized carriers are related both to the precise control of the chain length and monodispersity, due to the ability to seamlessly introduce precise modifications to their structures and biosynthesis at the genetic level. Genetically engineered polymeric drug carriers can be designed to assemble into nanoparticles or nanofibers. These nanostructures can be modified with multiple functional groups such as targeting moieties, imaging agents, or attachment sites for the purpose of drug and gene delivery. Similar to polymeric genetically engineered drug carriers, non-polymeric genetically engineered drug carriers such as vault proteins and viral proteins also form nanostructures, which are being explored for the delivery of genes and drugs. Delivery using these genetically engineered nanocarriers has yet to be translated aggressively to use in humans. At the current time, the understanding of these materials remains in its infancy. New ideas and perspectives are needed to advance genetically engineered nanocarriers into the clinic; however, their numerous preclinical applications suggest that they may provide a powerful new approach for creating nanomedicines.

\section{Acknowledgments}

This work was made possible by the University of Southern California, the National Institute of Health R21EB012281 to JAM, and P30 CA014089 to the Norris Comprehensive Cancer Center, the American Cancer Society IRG-58-007-48, the Stop Cancer Foundation, the USC Ming Hsieh Institute, and the USC Whittier Foundation.

\section{Disclosure}

JAM and PS are named as inventors on pending patent applications related to protein polymer nanomedicines. JAG has no conflicts of interest to disclose.

\section{References}

1. Langer R. Drug delivery and targeting. Nature. 1998;392(Suppl 6679): 5-10.

2. Rabotyagova OS, Cebe P, Kaplan DL. Protein-based block copolymers. Biomacromolecules. 2011;12(2):269-289.

3. Hecht S. Construction with macromolecules. Mater Today. 2005;8(3): 48-55.

4. Janib SM, Pastuszka MF, Aluri S, et al. A quantitative recipe for engineering protein polymer nanoparticles. Polym Chem. 2014;5(5):1614-1625.

5. MacKay J, Chen M, McDaniel JR, Liu W, Simnick AJ, Chilkoti A. Self-assembling chimeric polypeptide-doxorubicin conjugate nanoparticles that abolish tumours after a single injection. Nat Mater. 2009;8(12): 993-999.

6. Sun G, Hsueh PY, Janib SM, Hamm-Alvarez S, Andrew MacKay J. Design and cellular internalization of genetically engineered polypeptide nanoparticles displaying adenovirus knob domain. J Control Release. 2011;155(2):218-226.

7. Buehler DC, Toso DB, Kickhoefer VA, Zhou ZH, Rome LH. Vaults engineered for hydrophobic drug delivery. Small. 2011;7(10):1432-1439.
8. Koehl P, Delarue M. Polar and nonpolar atomic environments in the protein core: implications for folding and binding. Proteins. 1994;20(3): 264-278.

9. Elemans JAAW, Rowan AE, Nolte RJM. Mastering molecular matter. Supramolecular architectures by hierarchical self-assembly. J Mater Chem. 2003;13(11):2661-2670.

10. Urry DW. Physical chemistry of biological free energy transduction as demonstrated by elastic protein-based polymers. J Phys Chem B. 1997;101(51):11007-11028.

11. Valluzzi R, Winkler S, Wilson D, Kaplan DL. Silk: molecular organization and control of assembly. Philos Trans R Soc Lond B Biol Sci. 2002;357(1418):165-167.

12. Schellenberger V, Wang $\mathrm{CW}$, Geething $\mathrm{NC}$, et al. A recombinant polypeptide extends the in vivo half-life of peptides and proteins in a tunable manner. Nat Biotechnol. 2009;27(12):1186-1190.

13. Megeed Z, Cappello J, Ghandehari H. Genetically engineered silkelastinlike protein polymers for controlled drug delivery. Adv Drug Deliv Rev. 2002;54(8):1075-1091.

14. Georgens C, Weyermann J, Zimmer A. Recombinant virus like particles as drug delivery system. Curr Pharm Biotechnol. 2005;6(1):49-55.

15. Frandsen JL, Ghandehari H. Recombinant protein-based polymers for advanced drug delivery. Chem Soc Rev. 2012;41(7):2696-2706.

16. Urry DW, Urry KD, Szaflarski W, Nowicki M. Elastic-contractile model proteins: physical chemistry, protein function and drug design and delivery. Adv Drug Deliv Rev. 2010;62(15):1404-1455.

17. Cappello J, Crissman J, Dorman M, et al. Genetic engineering of structural protein polymers. Biotechnol Prog. 1990;6(3):198-202.

18. Shah M, Hsueh PY, Sun G, Chang HY, Janib SM, Mackay JA. Biodegradation of elastin-like polypeptide nanoparticles. Protein Sci. 2012;21(6):743-750.

19. Gustafson JA, Price RA, Greish K, Cappello J, Ghandehari H. Silkelastin-like hydrogel improves the safety of adenovirus-mediated genedirected enzyme-prodrug therapy. Mol Pharm. 2010;7(4):1050-1056.

20. Ferrari FA, Richardson C, Chambers J, et al, inventors; Protein Polymer Technologies Inc., assignee. Construction of synthetic DNA and its use in large polypeptide synthesis. United States patent US 5243038. September 7, 1993.

21. Aluri S, Pastuszka MK, Moses AS, MacKay JA. Elastin-like peptide amphiphiles form nanofibers with tunable length. Biomacromolecules. 2012;13(9):2645-2654.

22. Pastuszka MK, Janib SM, Weitzhandler I, Okamoto CT, Hamm-Alvarez S, MacKay JA. A tunable and reversible platform for the intracellular formation of genetically engineered protein microdomains Biomacromolecules. 2012;13(11):3439-3444.

23. Janib SM, Liu S, Park R, et al. Kinetic quantification of protein polymer nanoparticles using non-invasive imaging. Integr Biol (Camb). 2013;5(1):183-194.

24. Ravi S, Krishnamurthy VR, Caves JM, Haller CA, Chaikof EL. Maleimide-thiol coupling of a bioactive peptide to an elastin-like protein polymer. Acta Biomater. 2012;8(2):627-635.

25. Elia R, Newhide DR, Pedevillano PD, et al. Silk-hyaluronan-based composite hydrogels: a novel, securable vehicle for drug delivery. J Biomater Appl. 2013;27(6):749-762.

26. Numata K, Mieszawska-Czajkowska AJ, Kvenvold LA, Kaplan DL. Silk-based nanocomplexes with tumor-homing peptides for tumorspecific gene delivery. Macromol Biosci. 2012;12(1):75-82.

27. Numata K, Reagan MR, Goldstein RH, Rosenblatt M, Kaplan DL. Spider silk-based gene carriers for tumor cell-specific delivery. Bioconjug Chem. 2011;22(8):1605-1610.

28. Xia XX, Xu Q, Hu X, Qin G, Kaplan DL. Tunable self-assembly of genetically engineered silk - elastin-like protein polymers. Biomacromolecules. 2011;12(11):3844-3850.

29. Kim W, Xiao J, Chaikof EL. Recombinant amphiphilic protein micelles for drug delivery. Langmuir. 2011;27(23):14329-14334.

30. Amiram M, Quiroz FG, Callahan DJ, Chilkoti A. A highly parallel method for synthesizing DNA repeats enables the discovery of 'smart' protein polymers. Nat Mater. 2011;10(2):141-148. 
31. Simnick AJ, Amiram M, Liu W, et al. In vivo tumor targeting by a NGR-decorated micelle of a recombinant diblock copolypeptide. J Control Release. 2011;155(2):144-151.

32. Fluegel S, Buehler J, Fischer K, McDaniel JR, Chilkoti A, Schmidt M. Self-assembly of monodisperse oligonucleotide-elastin block copolymers into stars and compound micelles. Chemistry. 2011;17(20): 5503-5506.

33. Dreher MR, Simnick AJ, Fischer K, et al. Temperature triggered selfassembly of polypeptides into multivalent spherical micelles. $\mathrm{J} \mathrm{Am}$ Chem Soc. 2008;130(2):687-694.

34. García-Arévalo C, Bermejo-Martín JF, Rico L, et al. Immunomodulatory nanoparticles from elastin-like recombinamers: single-molecules for tuberculosis vaccine development. Mol Pharm. 2013;10(2):586-597.

35. Lewis RV. Spider silk: ancient ideas for new biomaterials. Chem Rev. 2006;106(9):3762-3774.

36. Pritchard EM, Kaplan DL. Silk fibroin biomaterials for controlled release drug delivery. Expert Opin Drug Deliv. 2011;8(6):797-811.

37. Numata K, Kaplan DL. Silk-based delivery systems of bioactive molecules. Adv Drug Deliv Rev. 2010;62(15):1497-1508.

38. Cappello J, Crissman JW, Crissman M, et al. In-situ self-assembling protein polymer gel systems for administration, delivery, and release of drugs. J Control Release. 1998;53(1-3):105-117.

39. Price R, Gustafson J, Greish K, Cappello J, McGill L, Ghandehari H. Comparison of silk-elastinlike protein polymer hydrogel and poloxamer in matrix-mediated gene delivery. Int J Pharm. 2012;427(1):97-104.

40. Farmer RS, Kiick KL. Conformational behavior of chemically reactive alanine-rich repetitive protein polymers. Biomacromolecules. 2005;6(3):1531-1539.

41. Dhandhukia J, Weitzhandler I, Wang W, MacKay JA. Switchable elastin-like polypeptides that respond to chemical inducers of dimerization. Biomacromolecules. 2013;14(4):976-985.

42. Shi P, Aluri S, Lin YA, et al. Elastin-based protein polymer nanoparticles carrying drug at both corona and core suppress tumor growth in vivo. J Control Release. 2013;171(3):330-338.

43. Shah M, Edman MC, Janga SR, et al. A rapamycin-binding protein polymer nanoparticle shows potent therapeutic activity in suppressing autoimmune dacryoadenitis in a mouse model of Sjogren's syndrome. J Control Release. 2013;171(3):269-279.

44. Gustafson JA, Ghandehari H. Silk-elastinlike protein polymers for matrix-mediated cancer gene therapy. Adv Drug Deliv Rev. 2010;62(15): $1509-1523$
45. Kar UK, Srivastava MK, Andersson A, et al. Novel CCL21-vault nanocapsule intratumoral delivery inhibits lung cancer growth. PloS One. 2011;6(5):e18758.

46. Han M, Kickhoefer VA, Nemerow GR, Rome LH. Targeted vault nanoparticles engineered with an endosomolytic peptide deliver biomolecules to the cytoplasm. ACS Nano. 2011;5(8):6128-6137.

47. Yildiz I, Shukla S, Steinmetz NF. Applications of viral nanoparticles in medicine. Curr Opin Biotechnol. 2011;22(6):901-908.

48. Wu Z, Chen K, Yildiz I, et al. Development of viral nanoparticles for efficient intracellular delivery. Nanoscale. 2012;4(11):3567-3576.

49. Pokorski JK, Steinmetz NF. The art of engineering viral nanoparticles. Mol Pharm. 2011;8(1):29-43.

50. Kedersha NL, Rome LH. Isolation and characterization of a novel ribonucleoprotein particle: large structures contain a single species of small RNA. J Cell Biol. 1986;103(3):699-709.

51. Suprenant KA. Vault ribonucleoprotein particles: sarcophagi, gondolas, or safety deposit boxes? Biochemistry. 2002;41(49):14447-14454.

52. Rome LH, Kickhoefer VA. Development of the vault particle as a platform technology. ACS Nano. 2013;7(2):889-902.

53. Berger W, Steiner E, Grusch M, Elbling L, Micksche M. Vaults and the major vault protein: novel roles in signal pathway regulation and immunity. Cell Mol Life Sci. 2009;66(1):43-61.

54. Goldsmith LE, Yu M, Rome LH, Monbouquette HG. Vault nanocapsule dissociation into halves triggered at low $\mathrm{pH}$. Biochemistry. 2007;46(10):2865-2875.

55. Galaway FA, Stockley PG. MS2 viruslike particles: a robust, semisynthetic targeted drug delivery platform. Mol Pharm. 2013;10(1): 59-68.

56. Choi KM, Choi SH, Jeon H, Kim IS, Ahn HJ. Chimeric capsid protein as a nanocarrier for siRNA delivery: stability and cellular uptake of encapsulated siRNA. ACS Nano. 2011;5(11):8690-8699.

57. Mackay JA, Chilkoti A. Temperature sensitive peptides: engineering hyperthermia-directed therapeutics. Int J Hyperthermia. 2008;24(6): 483-495.

58. Urry DW, Parker TM, Reid MC, Gowda DC. Biocompatibility of the bioelastic materials, poly(gvgvp) and its gamma-irradiation cross-linked matrix - summary of generic biological test-results. J Bioactive Compat Polymers. 1991;6:263-282.

59. Domb AJ, Kost J, Wiseman DM. Handbook of Biodegradable Polymers. Amsterdam: Harwood; 1997.
International Journal of Nanomedicine

\section{Publish your work in this journal}

The International Journal of Nanomedicine is an international, peerreviewed journal focusing on the application of nanotechnology in diagnostics, therapeutics, and drug delivery systems throughout the biomedical field. This journal is indexed on PubMed Central, MedLine, CAS, SciSearch ${ }^{\circledR}$, Current Contents ${ }^{\circledR} /$ Clinical Medicine,
Dovepress

Journal Citation Reports/Science Edition, EMBase, Scopus and the Elsevier Bibliographic databases. The manuscript management system is completely online and includes a very quick and fair peer-review system, which is all easy to use. Visit http://www.dovepress.com/ testimonials.php to read real quotes from published authors. 